\title{
Leer en la secundaria y leer en el primer año de psicología: perspectivas de los docentes con más y con menos experiencia
}

Reading at High School and Reading at First Year Psychology. More and Less Experienced Teachers' Perspectives

Leitura no ensino médio e leitura no primeiro ano de psicologia: perspectivas de professores com mais e menos experiência

María Micaela Villalonga-Penna* (iD orcid.org/0000-0002-0211-6870

\footnotetext{
Para citar este artículo: Villalonga-Penna, M. M. (2020). Leer en la secundaria y leer en el primer año de psicología: perspectivas de los docentes con más y con menos experiencia. Revista Colombiana de Educación, 1(80), 53-74. https://doi.org/10.17227/rce.num80-9591
}

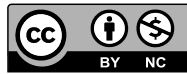

Recibido: 27/03/2019

Evaluado: 08/04/2020

* Doctora en Ciencias de la Educación. CONICET, Universidad Nacional de Tucumán, Argentina. Correo electrónico: mariamicaelavillalongapenna(a)conocet.gov.ar 


\begin{abstract}
Resumen
¿Cómo conciben la lectura los docentes del primer año de Psicología? ¿Reconocen diferencias entre las formas de leer en la escuela secundaria y en la universidad? Estos interrogantes guiaron el presente artículo de investigación en el cual abordamos las perspectivas de los docentes del primer año de la carrera de Psicología de una universidad pública en torno a estas cuestiones. En esta investigación de corte cualitativo y con diseño de teoría fundamentada entrevistamos a docentes con más de quince años y menos de diez en sus cargos. Analizamos las entrevistas a la luz de categorías preliminares predefinidas y también tuvimos en cuenta las emergentes. Los resultados dieron a conocer diferencias en las concepciones de ambos grupos de docentes sobre la lectura en contextos académicos y escolares, en lo que esperan de los estudiantes como lectores y en las razones a las que atribuyen las dificultades lectoras de estos. Así, conforme a la literatura consultada, los docentes con mayor experiencia conciben prácticas lectoras dialógicas y, en contraposición, los docentes con menor experiencia, prácticas lectoras monológicas. Mientras que los docentes más experimentados creen que es necesario enseñar a los estudiantes a leer en la universidad y reflexionar sobre las prácticas de enseñanza de la lectura, los menos experimentados tienden a atribuir las dificultades lectoras estudiantiles al secundario y la enseñanza de las habilidades necesarias para leer en la universidad a instancias de carácter remedial.
\end{abstract}

\section{Palabras clave}

lectura reflexiva; escuelas secundarias menores; psicología; profesores; representación del conocimiento

\section{Keywords}

reflexive reading; high schools; psychology;

teachers; knowledge representation

\begin{abstract}
How do first-year Psychology teachers conceive reading? Do they recognize differences between the ways of reading in high school and in college? These questions guided this research paper in which we approached the perspectives of teachers in the first year of the Psychology program of a public university on these issues. In this qualitative research with a grounded theory design we interviewed teachers with more than 15 and less than 10 years in their positions. We analyzed the interviews considering predefined preliminary categories, and we also took into account the emerging categories. The results showed differences in the conceptions of both groups of teachers about reading in academic and school contexts, in what they expect from their students as readers, and in the reasons they attribute to students' reading difficulties. Thus, according to the literature reviewed, teachers with more experience conceive dialogic reading practices; in contrast, teachers with less experience favor monological reading practices. While more experienced teachers believe that it is necessary to teach students to read at university and that it is necessary for teachers to reflect on their teaching practices, less experienced teachers tend to attribute students' reading difficulties to secondary school, and the teaching of necessary reading skills at the university

to instances remedial in nature.
\end{abstract}

\section{Resumo}

Como os professores do primeiro ano de Psicologia concebem a leitura? Eles reconhecem diferenças entre as formas de leitura no ensino médio e na faculdade? Essas questões nortearam este artigo de pesquisa em que abordamos as perspectivas dos professores do primeiro ano da carreira em Psicologia de uma universidade pública sobre essas questões. Nesta pesquisa qualitativa com desenho de teoria fundamentada, entrevistamos professores com mais de quinze anos e menos de dez em seus cargos. Analisamos as entrevistas à luz de categorias preliminares predefinidas e também levamos em consideração as emergentes. Os resultados revelaram diferenças nas concepções de ambos os grupos de professores sobre a leitura nos contextos acadêmico e escolar, no que esperam dos alunos como leitores e nos motivos pelos quais atribuem suas dificuldades de leitura. Assim, de acordo com a literatura consultada, professores com mais experiência concebem práticas de leitura dialógica e, em contrapartida, professores com menos experiência, práticas de leitura monológica. Enquanto professores mais experientes acreditam que é necessário ensinar os alunos a ler na faculdade e refletir sobre as práticas de ensino da leitura, os professores menos experientes tendem a atribuir as dificuldades de leitura dos alunos ao ensino médio e ao ensino das habilidades necessárias para ler na universidade a pedido de caráter corretivo.

\section{Palavras-chave}

leitura reflexiva; escola secundária; psicologia; professor; representação do conhecimento 


\section{Introducción}

¿Cómo conciben la lectura los docentes del primer año de la carrera de Psicología de una universidad pública? ¿Reconocen diferencias entre las formas de leer de la escuela secundaria y las de la universidad? ¿Qué función les asignan a las prácticas lectoras académicas? ¿Qué dificultades han detectado en los estudiantes en relación con la lectura? ¿Qué esperan de los estudiantes como lectores? Estos interrogantes han surgido en el curso de la presente investigación, en la cual buscamos describir las perspectivas acerca de la lectura que poseen los docentes del primer año de la carrera de Psicología de una universidad pública.

Las perspectivas docentes acerca de la lectura en las disciplinas y en contextos académicos han sido señaladas como un aspecto relevante ya que se encuentran relacionadas con lo que los maestros esperan de los estudiantes como lectores (Barton et al., 2000; Blommaert et al., 2007; Cassany, 2005; Lea, 2005; Lillis y Scott, 2007; Street, 2003), con la forma en que ellos declaran cómo organizan las prácticas lectoras en el aula, y con lo que desde la literatura de especialidad ha sido denominado la brecha o distancia entre las prácticas lectoras académicas y las que se desarrollan en la escuela secundaria (Eraut, 2000; Hermerschmidt, 1999; Lea, 2005; Lea y Street, 1998). En este sentido, en esta introducción presentamos una breve revisión de estudios centrados en los aspectos mencionados.

\section{Perspectivas y expectativas docentes en torno a la lectura en contextos académicos y escolares}

Sobre las perspectivas y expectativas de los docentes en torno a lectura, desarrollos internacionales describieron la distancia que puede existir entre las prácticas lectoras académicas y las del nivel educativo anterior, cuando los docentes presuponen que los estudiantes ya poseen los conocimientos lectores necesarios para encarar la lectura académica. En muchos casos, esto los lleva a esperar que los estudiantes se comporten como lectores autónomos capaces de autorregularse (Lea y Street, 1998). Esta clase de presuposiciones, según Lillis (1999), puede hacer que los docentes consideren innecesario explicitar pautas de lectura y los habilitaría a exigir cosas que no se han enseñado. En ambos casos, la literatura revisada coincide en señalar que las expectativas docentes pueden dificultar la permanencia en las instituciones universitarias de aquellos estudiantes menos familiarizados con las prácticas lectoras académicas (Collier y Morgan, 2008; Eraut, 2000; Lea y Street, 1997).

Desde esta perspectiva, las dificultades de los estudiantes se transforman en una suerte de déficit derivado de una incorporación asistemática de la lectura - en cuanto destreza generalizable a cualquier contexto educativo-

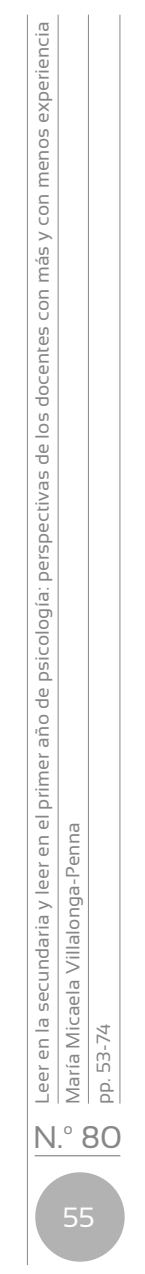


que no puede trasladarse o aplicarse desde el contexto escolar hacia los contextos académicos (Carlino y Estienne, 2004; Collier y Morgan, 2008; Falk Ross, 2002; Fernández y Carlino, 2010). Muchos autores cuestionaron esta clase de concepciones y estimaron que las convenciones lectoras propias de las culturas académicas deben enseñarse a los ingresantes para permitirles participar en la comunidad de referencia (Carlino, 2017; Carlino, 2019a; Cartolari y Carlino, 2016; Lea y Street, 1998; Lillis, 2001).

En nuestro país, teniendo en cuenta estos planteos, una investigación encarada por Carlino y Estienne (2004) caracterizó los modos de lectura esperados por los docentes en carreras del área de Humanidades y de Ciencias Sociales. Encontraron que los profesores esperaban que los alumnos leyeran los textos en función de los temas y de los objetivos del programa de la materia y según las perspectivas de las cátedras, jerarquizando determinadas cuestiones desarrolladas en los textos. Asimismo, los docentes esperaban que los estudiantes supieran que en ciencias humanas y sociales predominan los textos polifónicos, cuya comprensión hace necesario tener en cuenta diferentes perspectivas, muchas de las cuales pueden ser polémicas (Carlino y Estienne, 2004).

Consideraban a los estudiantes como sujetos capaces de autorregularse como lectores, lo cual, por un lado, los llevaba a no explicitar pautas sobre cómo leer los textos y, por el otro, a entender los pedidos de ayuda de los estudiantes como inmadurez y dependencia. Las autoras estimaron que estas cuestiones resultaban relevantes porque los alumnos eran evaluados en función de un modelo lector que no habían incorporado aún. Sin embargo, para algunos docentes el aprendizaje de la lectura disciplinar solo era factible con el acompañamiento de quienes ya forman parte de la cultura lectora universitaria (Carlino y Estienne, 2004).

\section{Funcionalidad de las prácticas lectoras en la universidad}

En relación con las concepciones acerca de lo que es leer en la universidad y la función de las prácticas lectoras académicas, una serie de desarrollos internacionales llevados a cabo principalmente por Dysthe et al. (Dysthe, 2011; Dysthe et al., 2006; 2012), y nacionales (Carlino, 2017; 2019b; Cartolari y Carlino, 2009; 2012; 2016) han propuesto dos maneras de concebir y organizar las prácticas de enseñanza y aprendizaje de la lectura: las monológicas y las dialógicas. En la misma dirección, investigaciones nacionales han identificado dos modalidades de construcción de las prácticas lectoras en el aula en educación superior: las periféricas y las entrelazadas (Carlino et al., 2010, Carlino et al., 2013).

Las formas monológicas se caracterizan por un predominio de las exposiciones orales magistrales por parte del docente en las cuales este puede aportar su interpretación de lo leído, con lo cual evita la discusión 
con los alumnos. Además, priman las comunicaciones organizadas por turnos determinados por el docente, que suelen no propiciar contribuciones sustantivas de los estudiantes al tema trabajado. En algunos casos, los docentes pueden llegar a evitar las interacciones verbales sobre lo leído o lo escrito por considerarlas poco productivas (Carlino, 2017; Carlino, 2019b; Cartolari y Carlino, 2012; 2016; Dysthe, 2011). En relación con esta forma de inclusión de las prácticas lectoras se encuentran las intervenciones "en los extremos" o "márgenes exteriores" de las actividades lectoras, en las que los docentes suelen dar una consigna a los estudiantes sin intervenir durante las actividades de lectura y corrigiendo las producciones de los alumnos al finalizar (Carlino et al., 2010; 2013).

Padilla et al. (2007; 2010; 2014) señalaron que en estas condiciones la palabra del docente puede llegar a ser la única legítima, lo que da lugar a la reproducción acrítica del conocimiento y a la acentuación de la dicotomía académica clase teórica (exposición magistral) frente a clase práctica (aplicación de conocimiento teórico).

Las formas dialógicas, en cambio, se caracterizan por un predominio de las interacciones entre los docentes y los alumnos basadas en preguntas que no se encuentran predefinidas. En estos casos, la lectura de los textos de la disciplina es uno de los propósitos de la materia y las clases son un espacio de construcción de significados e interpretaciones sobre los contenidos disciplinares basados en una multiplicidad de voces: la del docente, dirigida a focalizar los aspectos relevantes del tema; las de los alumnos y las de los autores. Se plantean así relaciones de intertextualidad entre el discurso oral de los intercambios en el aula y el discurso escrito presente en la bibliografía. En estas condiciones las interacciones en el aula en torno a los textos disciplinares facilitan el acceso de los estudiantes a los modos de leer y comprender en un área específica del conocimiento (Carlino, 2017; 2019b; Cartolari y Carlino, 2012; 2016; Dysthe, 2011). Vinculadas con esta forma de organización de las prácticas lectoras, se encuentran las entrelazadas, en las que los docentes trabajan mediando la lectura durante las clases e incentivando las interacciones con los alumnos para hablar sobre lo leído. La lectura opera como un medio para trabajar los temas de la materia, es decir, las tareas de lectura se entrelazan con el aprendizaje de contenidos conceptuales (Carlino et al., 2013).

\section{Diferencias entre las prácticas lectoras de la secundaria y las de la universidad}

Investigaciones nacionales revisadas profundizaron en las perspectivas docentes sobre la lectura en los niveles educativos antes citados (Fernández y Carlino, 2006; 2010). En una de ellas, Fernández y Carlino (2010) indagaron este aspecto en los primeros años de carreras de las áreas de 
Ciencias Humanas y Veterinarias de una universidad de gestión pública. Reconocieron dificultades en la comprensión y estimaron que la lectura en medios académicos es diferente a la de la secundaria, ya que se leen textos largos y complejos y es necesario establecer relaciones, no solo recordar ideas centrales. Consideraron que leer en la universidad en las dos áreas abordadas implicaba ingresar en un campo disciplinar específico y aprender el discurso especializado de este. Lo característico en Ciencias Humanas era que la lectura exigía aprender a relacionar los textos y los autores entre sí, y leer varias veces los textos y comprenderlos desde la perspectiva de una cátedra y de una comunidad disciplinar. La cantidad para leer marcaba otra diferencia porque hacía necesario que los alumnos pudieran autoorganizarse y aprender a expresar los planteos de los autores usando sus propias palabras (Fernández y Carlino, 2010).

En relación con lo anterior, estudios locales (Murga et al., 2002; Padilla, 2004) y nacionales (Carlino y Estienne, 2004; Fernández y Carlino, 2010) dieron a conocer que en la secundaria suelen privilegiarse visiones del conocimiento estables y válidas, que pueden dificultar la comprensión de la diversidad y los disensos inherentes al discurso científico-académico, especialmente en las ciencias sociales (Padilla, 2004). Además, suele predominar el uso de textos con un bajo nivel de complejidad y el trabajo con una única fuente textual, lo cual no posibilita la elaboración y organización de los conocimientos (Murga et al., 2002). En este sentido, la escuela secundaria de procedencia podría marcar una distancia en cuanto a la preparación con la cual llegan a la universidad (Fernández y Carlino, 2010).

\section{Metodología}

El problema de investigación planteado se ha trabajado primordialmente a partir de un enfoque cualitativo (Hernández Sampieri et al., 2008). La aproximación metodológica es la de una teoría fundamentada emergente (Glaser, 1992) ya que lo que se pretende es conocer el significado que los docentes han construido y construyen en relación con las prácticas lectoras académicas (Hernández Sampieri et al., 2008). Luego de realizar la inmersión inicial en el campo se optó por el diseño metodológico, se definieron el instrumento de recolección de datos y el tipo de muestreo.

\section{Instrumentos de recolección de datos}

Se realizaron entrevistas en profundidad a docentes a fin de conocer sus perspectivas acerca de la lectura académica en psicología y en asignaturas específicas. Durante la inmersión inicial se contactó y se tuvieron entrevistas muy breves con los docentes para dar a conocer el propósito de nuestra investigación y planificar el proceso. En estas sesiones, los 
maestros dieron a conocer las dificultades que detectaban en los alumnos como lectores y cómo muchas de ellas tienen que ver con la secundaria. En función de esto, y para la inmersión definitiva en el campo, el guion de entrevista contempló el abordaje de una serie de temas que permitieran ahondar en los que se mencionaron en el primer contacto: cómo ven a los estudiantes que ingresan a la carrera, en qué condiciones llegan los estudiantes al nivel universitario, cómo se lee en la secundaria, qué dificultades tienen los estudiantes en relación con la lectura, qué implica leer en la universidad, qué esperan de los alumnos como lectores académicos, de qué creen que dependen las posibilidades de los estudiantes de comprender exitosamente lo que leen en la universidad. Hemos entrevistado a 20 docentes del primer año de la carrera de siete asignaturas diferentes al iniciarse el ciclo lectivo.

\section{Muestreo intencional}

En este estudio participaron veinte docentes de siete asignaturas diferentes del primer año de la carrera de Psicología de una universidad pública. Luego de la inmersión inicial en el campo, estos participantes fueron divididos en dos grupos — de aquí en adelante G1 (grupo uno) y G2 (grupo dos) - en función de la antigüedad en sus cargos. En el G1 incluimos docentes que tenían más de quince años en sus cargos y en el G2, docentes con menos de diez. Tomamos este criterio ya que la antigüedad docente no se correspondía con ascensos en la carrera docente, pero sí con mayor experiencia en su práctica.

De los diez docentes del G1, uno era profesor titular de cátedra, cinco eran profesores adjuntos a cargo de cátedra y cuatro eran jefes de trabajos prácticos. De los diez docentes del G2, tres eran jefes de trabajos prácticos, seis eran auxiliares docentes de primera categoría y uno había obtenido recientemente el cargo de profesor adjunto a cargo de una materia — antes se desempeñaba como jefe de trabajos prácticos de esta-.

Los docentes entrevistados se seleccionaron teniendo en cuenta su antigüedad en los cargos docentes y su disponibilidad para participar en este estudio.

\section{Análisis de datos}

Luego de leer las transcripciones del primer contacto con los docentes, procedimos a elaborar el guion de entrevista. Entrevistamos a 5 docentes del G1 y a 5 del G2, transcribimos las entrevistas y realizamos la codificación inicial con base en las categorías que iban emergiendo. Obtuvimos 42 categorías iniciales. 
La codificación abierta dio a conocer como categorías: Guías de preguntas con sus respuestas, Resúmenes hechos por docentes, Manuales, Pocas hojas, Textos completos, Guías de estudio, Libros, Escasa anticipación en la lectura, Escasa cantidad para leer, Preeminencia de la voz docente, Fotocopias, Copias, Identificación de información específica en textos, Adquisición de información, Relación de fuentes discursivas, Construcción de conocimiento, Dificultades lectoras, Responsabilizar a la secundaria, Reflexionar sobre las prácticas de enseñanza, Docentes con mayor antigüedad, Docentes con menor antigüedad, Prácticas lectoras, Funcionalidad, Perspectivas, Alumnos, Docentes, Escuela, Secundaria, Facultad, Universidad, Brecha, Binomio, Enseñar, Aprender, Programas de materias, Fuentes, Una perspectiva, Múltiples perspectivas, Identificación de ideas centrales, Expectativas y Contextualizar. Algunas categorías resultaban redundantes: Fotocopias y Copias, Escuela y Secundaria; Universidad y Facultad. Por ello, optamos por la primera de la enumeración ya que semánticamente apuntaban a lo mismo. Otras, se encontraban en la misma línea semántica: Leer desde una perspectiva y Leer para adquirir información específica; Leer desde múltiples perspectivas y Leer para construir conocimientos. Nos centramos en la primera denominación.

Posteriormente, refinamos las categorías, y para ello, comparamos cada fragmento con los ubicados previamente en la misma categoría a fin de obtener cierta coherencia interna. En ambos procesos la comparación constante de los diferentes segmentos y categorías nos permitió encontrar similitudes y diferencias entre categorías. En este proceso obtuvimos quince categorías. A partir de allí, entrevistamos a los cinco docentes restantes de cada grupo buscando indagar para cada grupo las distancias percibidas entre las prácticas lectoras de la secundaria y las de la universidad, las prácticas lectoras de la universidad y las expectativas docentes.

Refinamos aún más las categorías y quedaron establecidas las que se presentan en los resultados. Además, cuando no encontramos nuevas categorías y los datos se tornaban redundantes, consideramos que se saturaron las categorías y detuvimos la recolección y el análisis de datos. De este modo, quedaron establecidos cuatro grandes temas que aúnan las categorías citadas y que dan cuenta de la discrepancia entre las perspectivas de los docentes con más y con menos experiencia: la contraposición entre las prácticas lectoras de la secundaria y las de la universidad, la contraposición entre dos prácticas lectoras académicas (Leer para adquirir información específica y Leer para aprender), la contraposición acerca del origen de las dificultades lectoras de los estudiantes, y las expectativas docentes sobre los alumnos como lectores. Consideramos como categoría eje o central la referida a las perspectivas sobre la funcionalidad de la lectura. En torno a ella se manifiestan las discrepancias entre la secundaria y la universidad, las perspectivas de los docentes con más y con menos experiencia, y en 
relación causal con estas dos, la atribución del origen de las dificultades lectoras de los estudiantes y las expectativas de los docentes en torno a las prácticas lectoras.

Para el análisis nos valimos del Atlas.ti (versión 6).

\section{Resultados}

Del análisis de las entrevistas a docentes con mayor y con menor experiencia se derivan básicamente cuatro grandes temas que abarcan una serie de categorías:

\section{Perspectivas sobre la funcionalidad de la lectura en la secundaria: leer en la secundaria vs leer en la universidad}

Lo primero que se desprende del discurso de los docentes del G2 son ciertas prácticas de lectura propias de la escolaridad secundaria y las clases de textos vinculadas a ellas: el dictado de guías con sus respuestas (por parte de los docentes) y la lectura de resúmenes de los temas hechos por los profesores. En cuanto al tipo de textos, indican que los estudiantes no leen de libros de texto, o si leen, se trata solo de unas pocas hojas del manual. Manifiestan que este tipo de prácticas les permitía a los estudiantes aprobar exámenes en ese nivel educativo. También, apreciamos las diferencias con las prácticas lectoras propias de la universidad (leer textos completos, responder a preguntas de guías de estudio, aprobar exámenes parciales).

D: Lo que pasa es que en el secundario muchas veces trabajan, según lo que me cuentan los chicos, con guías que los docentes les dictan y eso estudian para las pruebas, o ven los temas de resúmenes que les hacen los profes o ven un par de hojitas de un manual y nada más. Y aquí las guías tienen que resolverlas ellos y se leen textos completos, por cortos o largos que sean. (Docente 10, G2)

E: Cuando vos me decís que no saben estudiar ¿a qué te referís?

D: Estrategias, herramientas, creo que no les enseñan a estudiar, no les enseñan técnicas de estudio en el colegio. Ni tampoco los hábitos de estudio, eso de sentarse e ir dosificando los temas a estudiar día a día... La vez pasada... una alumna... me dice que estaban acostumbrados a que la profesora en el colegio les daba la guía hecha y que ellos la estudiaban y la rendían. Me decía que ellos no tenían que buscar las respuestas en ninguna parte, que no trabajaban con libros de texto. (Docente 9, G2)

Además, del discurso de los docentes del G1 se infiere que las prácticas implican un uso del tiempo que se vincula con la cantidad a leer (un binomio tiempo/cantidad), con el rol del docente, con las clases textuales 
y con una práctica específica de lectura. Así, apreciamos la preeminencia del discurso docente, la escasa anticipación con la que se lee en la secundaria, la palabra del docente como la única autorizada, la escasa cantidad de material para leer (en contraste con el volumen que se debe leer en la universidad), el hecho de que los alumnos parecen centrarse más en la cantidad que en la comprensión, el uso de fotocopias o manuales y la identificación de información específica en los textos (el párrafo que responden a la pregunta). Solo una profesora comenta que también es docente en la secundaria.

E: A ver, ¿entonces vendría a ser como que ustedes han observado que hay ciertas prácticas que tienen que ver con las estrategias de lectura que han desarrollado que no facilitarían la comprensión del material bibliográfico?

D: Si... solamente mirar el cuadernillo ya los impacta.

E: ¿El tamaño?

D: Sííí... en la secundaria el profesor entrega una fotocopia para trabajar en el momento o cuando leen el manual son tres o cuatro hojitas... siempre son textos cortos... por ahí lo que está como focalizado es en eso... la cantidad, más que en la comprensión del texto... y a veces los docentes del secundario, además enfatizan esto de identificar el concepto principal o el párrafo que responde a la pregunta $X$ de la guía, pero desgajado del resto del texto... sin la contextualización general. Y entonces de ahí aparece la idea del resumen y memorístico... Es como un copiar y pegar acrítico. Además está eso de que los conocimientos se impartan desde otro, que otro les indique que es lo importante del texto y no importa que no les diga por qué. (Docente 2, G1)

En este sentido, notamos que tanto los docentes con mayor experiencia como aquellos con menor experiencia dan a conocer las distancias $O$ brechas entre las prácticas lectoras de la secundaria y las de la universidad por lo que se plantea la relación de oposición leer en la secundaria versus leer en la universidad. A su vez, estas categorías se incluyen en una más amplia referida a qué perspectivas tienen los docentes sobre la función de la lectura en la secundaria.

\section{Perspectivas sobre la funcionalidad de la lectura en la universidad: adquirir información vs construir conocimientos disciplinares}

En las perspectivas de los docentes del G1 sobre la lectura en contextos académicos, en el discurso docente apreciamos dos posturas contrapuestas: por un lado, los docentes que acentúan como función de la lectura la adquisición de información. 
E: ¿Qué espera que hagan sus alumnos al leer?

D: Mmmm... que puedan buscar, recortar esa información específica, que ya se la anticipó en la clase teórica. Porque en esta materia, la teórica les indica a los chicos la profundidad con la cual tienen que leer el texto y lo que realmente es importante. (Docente 1)

Por el otro, los docentes que indican como función de la lectura la construcción de conocimientos disciplinares.

E: Y vos, ¿qué función creés que puede tener o cumplir la lectura del material bibliográfico que da la cátedra?

D: Si vos fomentás que los chicos lean buscando solamente el concepto así, aislado... los chicos van a buscar eso, información específica. Pero si les mostrás que esa información específica no tiene sentido si está aislada, y que la única forma de entender eso que es relevante es teniendo en cuenta las ideas que viene proponiendo el autor y quién escribe, por qué escribe, en qué momento escribe... entonces, ahí sí se puede hablar de construir conocimientos. Ahí se puede hablar también de poder relacionar lo que me dice un autor con lo que me dice otro autor, relacionar lo que leo en la fuente primaria con lo que leo en la fuente secundaria, y esto de relacionar es lo que permite a los chicos ir construyendo, armando, aprendizajes, conocimientos [se ríe]. Y entonces leer es la vía que permite esto y por eso se vuelve fundamental.

(Docente 4, G1)

Por su parte, los docentes del G2 remarcan como función de la lectura la adquisición de información.

D: Eso que te decía, que busquen esos conceptos que se dieron en la teórica, que los busquen en el texto porque eso es lo importante... eso es lo que les van a tomar en el parcial. (Docente 6, G2)

En este caso, inicialmente pueden apreciarse en el grupo de los docentes con mayor experiencia dos maneras contrapuestas de concebir las prácticas lectoras: leer para adquirir información específica y leer para aprender contenidos disciplinares. Luego, puede notarse que los docentes con menor experiencia parecen concebir la lectura como medio para adquirir información. Es por esto que las dos categorías se incluyen en un tema más amplio referido a las perspectivas sobre qué función tiene la lectura en la universidad para estos docentes. Asimismo, y como veremos, estas categorías resultan semánticamente afines con las que conciben la lectura académica desde una o desde múltiples perspectivas. 


\section{Perspectivas docentes sobre las dificultades lectoras de los alumnos: Atribuirlas a la secundaria o reflexionar sobre las prácticas de enseñanza}

En general, los docentes del G2, al hablar de dificultades lectoras de los estudiantes, las vinculan con cosas que no se habrían enseñado en la secundaria (la base de la secundaria), con el binomio tiempos/cantidad (el no leer y el no poder organizar los tiempos en función de la cantidad a leer) y las prácticas de lectura (problemas para identificar ideas principales en un texto o resumir porque marcan todo).

Son estas cuestiones las que marcarían también una distancia con las prácticas de lectura en la universidad.

E: Y en relación a la lectura ¿has identificado alguna dificultad específica?

D: Sí [se ríe], no leen. O leen y subrayan todo, o no leen y no subrayan nada. No todos vienen de una buena secundaria... o pretenden leer en un día una cantidad bien importante de material, y no llegan... (Docente 8, G2)

Los del G1 relacionan las dificultades con las fuentes textuales (la presencia de fuentes primarias y la escasez de fuentes secundarias), la complejidad de los textos por leer (textos enrevesados), la identificación de ideas principales, la falta de lectura (cuadernillos en blanco). También dan a conocer que estas dificultades se relacionarían con lo que no ha sido enseñado en la secundaria en relación con la lectura.

D: [...] está la constante: los cuadernillos en blancos, [sic] la dificultad para elegir ideas principales y secundarias, dificultad como para recortar conceptos centrales en los textos, la profundidad con que estudian los conceptos, el nivel de aprehensión de los conocimientos, de los conceptos. Hay una dificultad en esto, y tiene que ver con que en el secundario, no les enseñaron a leer. (Docente 5, G1)

E: Algo hablamos sobre la bibliografía pero ¿qué desafíos creés que tendrán los chicos para leer textos de Psicología?

D: En esta materia, lo que te decía, la cantidad abrumadora de fuentes primarias y la escasez de fuentes secundarias. La cantidad a leer. Los temas que se tratan en la materia, la complejidad y profundidad con que hay que abordarlos. Esas cosas pueden ser difíciles para los chicos y también para mí. Hay textos que explican cosas de manera sencilla y directa, hay otros más enrevesados, como los freudianos [...] El tema es que, suelen tener dificultades para separar ideas principales de ideas secundarias [...]. (Docente 3, G1) 
El discurso de los docentes del G2, da a conocer que si bien no se puede responsabilizar totalmente a la escolaridad secundaria por las dificultades lectoras de los estudiantes, la base o formación es mala o no provee las herramientas necesarias.

D: $Y$ creo que no se puede culpar al estudiante si lee y no entiende...

E: ¿Por qué?

D: Porque es cierto que muchas veces vienen con una mala base del secundario, hay cosas que no les enseñaron...por ejemplo a extraer ideas principales de textos, a no perderse en el bosque. (Docente 1, G1)

A diferencia de los del G2, el discurso de los docentes del G1 permite entrever dos aspectos: por una parte, que la institución universitaria se ve en la obligación de enseñar lo que la secundaria no enseñó y, por la otra, que aunque la base de la secundaria puede llegar a determinar algunas cosas, también existe la posibilidad de aprender a leer en la universidad.

E: Y, estas habilidades o estrategias de lectura y de estudio ¿usted cree que el secundario debió enseñarlas o que se pueden enseñar en la universidad?

D: Yo pienso que es responsabilidad de la secundaria. Pero, que la universidad se ha visto obligada a hacerse cargo, se ha visto obligada. Y en ese sentido se ha bajado el nivel de exigencias en algunos aspectos.

(Docente 4, G1)

Inicialmente tanto los docentes con mayor como con menor experiencia identifican qué aspectos relacionados con la lectura académica representan una dificultad para los estudiantes. Después, relacionan estas dificultades con lo que el nivel educativo anterior no enseñó, y solo los docentes con mayor experiencia reflexionan sobre la necesidad de enseñarlo. Por esto se plantea la dicotomía entre las formas de concebir las dificultades lectoras: atribuirlas de manera causal a la secundaria y desligarse de toda responsabilidad, en contraposición a asumir la responsabilidad de enseñar a leer en la universidad.

\section{Expectativas docentes acerca de los alumnos como lectores: Leer desde una sola perspectiva versus leer desde múltiples perspectivas}

En cuanto a las expectativas docentes, estas tienen que ver con las prácticas de lectura estudiantiles. Inicialmente, tanto los del G1 como los del G2 reconocen que hay cosas que no pueden esperar que los estudiantes hagan. Pero mientras que los del G1 apuntan a enseñar estas cosas, los del G2, optan por delegar esa función a los espacios de tutoría institucional. 
D: Yo no puedo esperar que hagan cosas que no he enseñado yo y bueno, ya sabemos que hay cosas que hubiera estado bueno que aprendan en el secundario, pero si no las han aprendido, no podemos esperar que hagan cosas que no van a hacer... hay que enseñarles. (Docente 2, G1)

D: Lamentablemente, lo que no se aprendió en el secundario aquí yo no puedo enseñarlo y pero bueno, está el SITA, ${ }^{1}$ para que puedan aprender eso que no les enseñaron antes... pero además de eso yo ya sé que no puedo esperar cosas que no pueden hacer...por eso creo que hemos bajado el nivel de exigencias. (Docente 7, G2)

Además, los enseñantes del G1 son principalmente quienes plantean una práctica lectora que sería deseable: leer y considerar los programas de las materias. Al respecto, expresan dos situaciones disímiles: por un lado, el hecho de que muchos estudiantes desconocen que existen los programas de las materias y, por el otro, la necesidad de que los estudiantes aprendan a leer teniendo en cuenta el programa como guía.

D: Mirá, para empezar pocos saben que tienen el programa de la materia...

E: ¿Y lo principal, las ideas principales, en función de qué se determinan o se buscan?

D: Y de pronto, si nos vamos a poner a ver... mirá si te pones a ver hay programas de las materias y ahí en el programa está lo que nos importa para enfocar los textos... y es lo que también se ve en las teóricas. (Docente 1, G1)

Finalmente, se plantea una contradicción que retoma las anteriores: leer desde una sola perspectiva (la de los docentes) y leer desde múltiples perspectivas (es decir, enlazando diferentes discursos). Así, los docentes del G2 esperan que los estudiantes lean en función de lo que ellos marcan como ideas centrales que deben identificar en los textos.

D: Y bueno, a veces pasa que vos leés un texto para una materia y recortás esas ideas principales en función de lo que te pide un profe... y años después lo leés al mismo texto en una materia y recortás otras ideas principales y entonces es como si descubrieras otra versión del texto... pero lo cierto es que nosotros los profes también esperamos que los alumnos vean esas ideas que nos parecen importantes.

(Docente 10, G2)

Por su parte, y a diferencia de los docentes del G2, los del G1 buscan que sus alumnos no solo identifiquen ideas centrales del texto, sino también que puedan contextualizar lo que leen y relacionar las explicaciones del profesor o lo que dicen otros autores con lo que están leyendo. 
D: Me importa mucho que sepan quién escribió el texto, por qué, en qué momento específico... que sepan si lo que leen es un capítulo de libro, o un artículo o una ficha de cátedra... que puedan dimensionar si tienen la primera, la segunda o que número de edición... para que puedan de alguna forma situarse, contextuar eso que leen, que vean que no son cosas aisladas que se estudian caprichosamente para aprobar tal o cual materia...que esto que dice un autor va más allá de, lo que nosotros les pedimos que estudien para nuestra materia... también me importa que puedan identificar ideas centrales de un texto, pero no de forma aislada, sino contextuando y relacionando eso que está en el texto con las explicaciones del profesor o con lo que dicen otros textos. (Docente 3, G1)

Tanto los docentes con mayor experiencia como aquellos con menor experiencia coinciden en que durante la escolaridad secundaria los estudiantes leen fuentes secundarias en las que la versión del conocimiento que presenta el docente es la que posee mayor legitimidad. Mientras que los docentes con menos experiencia se centran en la diferencia en el nivel de exigencias entre ambos niveles educativos, los que tienen mayor experiencia remarcan las distancias entre las prácticas lectoras estudiantiles y la necesidad de reflexionar sobre las prácticas pedagógicas.

Los docentes con mayor experiencia proponen la lectura académica como una herramienta para construir conocimientos disciplinares y para adquirir información específica. En este sentido, plantean la necesidad de leer y considerar los programas de las materias y buscan que sus alumnos no solo identifiquen ideas centrales del texto, sino también, que puedan contextualizar lo que leen y relacionar las explicaciones del profesor o lo que dicen otros autores con lo que están leyendo. En contraposición, los docentes con menor experiencia acentúan solo la adquisición de información, es decir, esperan que los estudiantes lean en función de lo que ellos marcan como ideas centrales que deben identificar en los textos. La práctica lectora planteada por ambos grupos que se centra en la adquisición de información se sitúa en la misma línea que las prácticas lectoras que ellos describen como propias de la escolaridad secundaria. En este sentido, solo algunos docentes con más experiencia parecen notar genuinamente la distancia que se genera entre las formas de lectura de la secundaria y las de la universidad y la necesidad de revisar las prácticas de enseñanza.

A pesar de que ambos grupos de docentes proponen prácticas lectoras que se presentarían como una prolongación de las enseñadas y aprendidas en la secundaria - la lectura como medio para la adquisición de información específica-, indican que este no proveería las herramientas lectoras necesarias para afrontar los desafíos de la lectura académica, lo cual constituye una contradicción. Sin embargo, el hecho de que los docentes con mayor experiencia propongan la necesidad de reflexionar

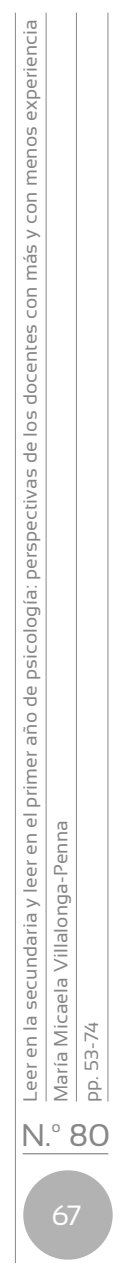


sobre las practicas pedagógicas y de asumir la explicitación y la enseñanza de prácticas lectoras propias de los contextos académicos daría cuenta de cierto nivel de concientización sobre la brecha que se genera entre ambos niveles educativos y cómo no se puede exigir ni esperar que los recién llegados a una comunidad disciplinar hagan cosas que no se han enseñado. En contraste, los docentes con menor experiencia no se proponen enseñar lo que los estudiantes non saben en relación con la lectura, sino delegar esto en instancias que parecen tener un carácter remedial (espacios de tutoría institucional).

\section{Discusiones}

El análisis de las entrevistas nos muestra perspectivas y expectativas distintivas de los docentes con mayor y con menor experiencia en relación con la lectura en esta comunidad académica. Sobre las perspectivas acerca de la lectura en la secundaria y en la universidad, los docentes de ambos grupos identifican diferencias entre las prácticas lectoras en los dos niveles educativos. Los docentes tienden a definir las prácticas lectoras de la secundaria estableciendo un contrapunto disyuntivo con las de la universidad; con base en este contraste, parecen indicar como función de la lectura y como práctica social legitimada la adquisición de información específica que es señalada como relevante por los docentes. Además, ambos grupos de docentes coinciden en que se leen clases de textos y cantidades que en algunos casos resultan afines a los descritos en la literatura revisada, esto es, textos con un bajo nivel de complejidad, escasa cantidad para leer, poca anticipación y un texto por tema de estudio (Carlino, 2019a; Carlino y Estienne, 2004; Fernández y Carlino, 2010). Sin embargo, los docentes con menos experiencia identifican una práctica no descrita en la literatura revisada: el dictado de guías con sus respuestas y de resúmenes sobre los temas, que constituyen lo que podríamos llamar "textos simplificados"; esto no favorecería una construcción auténtica de sentido por parte de los estudiantes, ya que la única voz autorizada sería la del docente (Douglas et al., 2011; Padilla, 2004) quien sesga lo significativo del tema por trabajar.

Lo anterior coincide con lo indicado por Padilla (2004) acerca del tipo de prácticas lectoras que se construyen en este nivel educativo, que se caracterizan por una visión del conocimiento estable, válida y poco discutible. Tal y como lo plantea la citada autora, esto pareciera dificultar la comprensión de la diversidad, la precariedad y los disensos inherentes al discurso científico-académico en las Ciencias Sociales.

Sobre la funcionalidad de la lectura en la universidad, para los docentes con mayor experiencia la lectura es un medio o una herramienta para adquirir información y para construir conocimientos disciplinares. Esto 
resulta coincidente con lo propuesto por Dysthe et al. (Dysthe, 2011; 2012; Dysthe et al., 2006) y por Carlino et al. (Carlino, 2017; 2019a; Carlino, 2019b; Carlino et al., 2010; Cartolari y Carlino, 2009; 2012; 2016; Cartolari et al., 2010; Padilla y Carlino, 2010) quienes han identificado modalidades afines de concepción y construcción de prácticas lectoras académicas. Así, los docentes con mayor experiencia plantean, al igual que los desarrollos acerca de la dialogicidad de las prácticas lectoras académicas (Carlino, 2017; Cartolari y Carlino, 2012; 2016; Dysthe, 2011; 2012; Dysthe et al., 2006), la vinculación o el entrelazamiento (Carlino et al., 2013) entre las explicaciones docentes dadas en las clases teóricas, lo propuesto por los autores en los textos, la necesidad de contextualizar lo que se lee y la lectura de diversas fuentes textuales. A diferencia de estos docentes, los que tienen menos experiencia consideran que la lectura es un medio para adquirir la información que el docente estima importante y que garantizaría aprobar los exámenes, lo cual da cuenta de la preeminencia de la palabra docente o monologicidad (Carlino, 2017; Cartolari y Carlino, 2012; 2016; Dysthe, 2011; 2012; Dysthe et al., 2006).

Acerca de las expectativas docentes, los que tienen menos experiencia esperan que los estudiantes puedan leer y recortar o adquirir información específica de los textos con lo cual sus expectativas apuntarían a prácticas lectoras de tipo monológico (Dysthe, 2012; Dysthe et al., 2006). En cambio, los que poseen más experiencia, esperan que los estudiantes sean capaces de leer según las perspectivas de las cátedras, en función de lo que piden los profesores y con la antelación necesaria, lo cual resulta afín a los hallazgos nacionales (Carlino y Estienne, 2004; Fernández y Carlino, 2010). Además, esperan que los estudiantes lean buscando ideas centrales, relacionando las explicaciones del docente con la información de los textos y con sus conocimientos previos, contextualizando los textos. También esperan que los estudiantes puedan leer en función del programa de la materia, lo cual se sitúa en la misma dirección que lo indicado en investigaciones nacionales e internacionales sobre las prácticas lectoras dialógicas (Carlino y Estienne, 2004; Carlino et al., 2013; Dysthe, 2012; Dysthe et al., 2006; Fernández y Carlino, 2010; Padilla et al., 2005; Padilla et al., 2010).

Ambos grupos señalan que las dificultades en torno a la lectura derivan de la secundaria, pero solo los docentes con mayor experiencia proponen que la universidad puede enseñar las convenciones propias de la lectura académica. Esto último se sitúa en la misma dirección que la literatura revisada, que considera que las dificultades lectoras no son un déficit que los alumnos deban resolver por sí solos porque el ingreso a la universidad supone nuevos roles lectores que los ingresantes provenientes de otras culturas académicas desconocen (Carlino, 2019a, 2019b; Carlino y Estienne, 2004; Collier, 2000; Collier y Morgan, 2008; 
Hermerschmidt, 1999). Además, cabe señalar la perspectiva de aquellos docentes que parecen asignar la enseñanza de la lectura a instancias de tipo remedial.

En suma, este estudio nos muestra las distancias percibidas por los docentes entre las prácticas lectoras del nivel secundario y las de la universidad en esta comunidad académica. Los dos grupos participantes tienden a describir las prácticas lectoras de la secundaria como monológicas. Sin embargo, al dar cuenta de las prácticas lectoras académicas, los docentes con menos experiencia ponen el acento en la lectura como un medio para la adquisición de información y los que tienen más experiencia, en la construcción de conocimientos disciplinares con base en la lectura. Además, si bien ambos grupos de docentes explicitan el contrapunto entre las prácticas lectoras de estos niveles educativos, solo una docente manifiesta que trabaja en el nivel educativo secundario con lo cual cabría cuestionar si la forma de concebir las prácticas lectoras escolares resulta afín con cómo se desarrollan estas en las aulas de la secundaria.

En relación con las concepciones sobre la lectura en la universidad, se encuentran las expectativas que tienen los profesores sobre sus estudiantes como lectores. En esta comunidad académica y disciplinar, los docentes con mayor experiencia parecen esperar que los estudiantes desplieguen prácticas lectoras dialógicas (leer en función de un programa de estudios y leer contextualizando) o entrelazadas, y los menos experimentados, prácticas lectoras monológicas (leer en función de lo que piden los profesores y recortando información específica). Ambos grupos parecen esperar que los estudiantes se comporten como lectores autónomos (Carlino y Estienne, 2004; Eraut, 2000; Hermerschmidt, 1999). Teniendo en cuenta nuestros hallazgos y los de estudios internacionales (Collier y Morgan, 2000; Eraut, 2000; Lea y Street, 1998) cabe preguntarse si los docentes pueden exigir a los estudiantes universitarios cosas que no se les enseñaron, y si esto obstaculizaría el acceso y la permanencia de los estudiantes menos familiarizados con las culturas académicas (Collier y Morgan, 2000; Eraut, 2000).

La principal contribución de este estudio radica en haber dado a conocer que en esta comunidad académica hay una distancia entre las perspectivas acerca de la lectura de los docentes con mayor y con menor experiencia y que dichas concepciones los llevan a esperar de los estudiantes cosas diferentes. Por una parte, los primeros conciben prácticas lectoras dialógicas, esperan que los estudiantes lean de dicho modo y reflexionan acerca de la posibilidad de la universidad de enseñar estas cuestiones a los noveles lectores académicos y de ayudar a aquellos que posean dificultades. Por la otra, los segundos conciben prácticas lectoras monológicas, esperan que los estudiantes lean de este modo, y tienden a 
atribuir las dificultades lectoras estudiantiles a la secundaria y la enseñanza de las habilidades necesarias para leer en la universidad a instancias de carácter remedial.

Esto nos lleva a preguntarnos por qué los docentes más experimentados han construido esta visión acerca de su rol de enseñantes, de qué modo han participado y participan en las prácticas de enseñanza de la lectura en esta comunidad académica y disciplinar, y por qué los docentes menos experimentados no conciben las prácticas lectoras de manera similar. Creemos que esto ameritaría continuar profundizando el análisis de las perspectivas y expectativas docentes de ambos grupos. También nos cuestionamos si lo declarado por los docentes se verifica en la práctica y si coincide o no con las perspectivas de los estudiantes acerca de la lectura académica, cuestiones que podrían abordarse en estudios posteriores. Finalmente, como se trata de una investigación de corte cualitativo y la muestra resulta reducida desalentamos la realización de generalizaciones más allá de casos similares en circunstancias afines.

\section{Referencias}

Barton, D., Hamilton, M., e Ivanič, R. (2000). Situated literacies: Reading and writing in context. Psychology Press.

Blommaert, J., Street, B., Turner, J. y Scott, M. (2007). Academic literacies: What have we achieved and where to from here. Journal of Applied Linguistics, 4(1), 137-149.

Carlino, P. (2003). Leer textos complejos al comienzo de la educación superior: tres situaciones didácticas para afrontar el dilema. Textos. Didáctica de la Lengua y la Literatura, 33, 43-51.

Carlino, P. (2017, febrero). Aprender en la universidad: ¿qué diferencia hace si se lee, se escribe y se habla sobre lo leído y escrito? [ponencia]. VIII Encuentro "Experiencias significativas en lectura y escritura". Cali, Colombia.

Carlino, P. (2018). Dos variantes de la alfabetización académica: cuando se entrelazan la lectura y la escritura en las materias. Signo y Pensamiento, 36(71), 16-32. https://doi.org/10.11144/Javeriana.syp36-71.dvaa

Carlino, P. (2019a). Escribir, leer y aprender en la universidad. Una introducción a la alfabetización académica. Fondo de Cultura Económica.

Carlino, P. (2019b). Dos variantes de la alfabetización académica [ponencia]. ıx Congreso Internacional de la Cátedra Unesco para el Mejoramiento de la Calidad y Equidad de la Educación en América Latina, con Base en la Lectura y la Escritura. Cuenca, Ecuador. Julio del 2018. 
Carlino, P. y Estienne, V. (2004). ¿Pueden los universitarios leer solos? Un estudio exploratorio. Memorias de las xı Jornadas de Investigación en Psicología. Facultad de Psicología de la Universidad de Buenos Aires, 29, 174-177.

Carlino, P., Iglesia, P. y Laxalt, I. (2010, 9-10 de agosto). Leer y escribir en la formación de profesores secundarios de diversas disciplinas: qué dicen los docentes que se hace [ponencia]. Jornadas Nacionales Cátedra Unesco de Lectura y Escritura Lectura, escritura y aprendizaje disciplinar.

Carlino, P., Iglesia, P. y Laxalt, I. (2013, julio). Qué dicen los docentes que hacen cuando los alumnos no comprenden lo que leen [ponencia]. XII Congreso Latinoamericano para el Desarrollo de la Lectura y la Escritura. Iv Foro Iberoamericano de Literacidad y Aprendizaje. Puebla, México.

Cartolari, M. y Carlino, P. (2009, noviembre). Formación docente, concepciones y prácticas de lectura y escritura: una revisión bibliográfica [ponencia]. । Congreso Internacional de Investigación y Práctica Profesional en Psicología xvı Jornadas de Investigación. Quinto Encuentro de Investigadores en Psicología del Mercosur. Universidad de Buenos Aires.

Cartolari, M. y Carlino, P. (2012). Leer y tomar apuntes para aprender en la formación docente: un estudio exploratorio. Magis. Revista Internacional de Investigación en Educación, 4(7).

Cartolari, M. y Carlino, P. (2016). Hacerle lugar o no a la lectura en aulas del nivel superior: experiencias contrastantes en dos materias de un profesorado en Historia. En G. Bañales Faz, M. Castelló Badía y N. Vega López (comps.), Enseñar a leer y a escribir en la educación superior (pp. 160-180). sM México y Universidad Autónoma de Tamaulipas.

Cartolari, M., Carlino, P. y Rosli, N. (2010). Hacerle lugar o no a la lectura en la formación docente en ciencias sociales [ponencia]. Jornadas Nacionales de la Cátedra Unesco de Lectura y Escritura "Lectura, escritura y aprendizaje disciplinar". Septiembre del 2009.

Cassany, D. (2005). Literacidad crítica: leer y escribir la ideología [taller]. Ix Simposio Internacional de la Sociedad Española de Didáctica de la Lengua y la Literatura.

Collier, P. J. y Morgan, D. L. (2008). Is that paper really due today?: Differences in first-generation and traditional college students' understandings of faculty expectations. Higher Education, 55(4), 425-446.

Dysthe, O. (2011). Opportunity spaces for dialogic pedagogy in test-oriented schools: A case study of teaching and learning in high school. Bakhtinian Pedagogy: Opportunities and Challenges for Research, Policy and Practice in Education across the Globe, 90, 69. 
Dysthe, O., Bernhardt, N. y Esbjørn, L. (2012). Dialogue-based teaching. The Art Museum as a learning space. Skoletjenesten.

Dysthe, O., Samara, A. y Westrheim, K. (2006). Multivoiced supervision of master's students: A case study of alternative supervision practices in higher education. Studies in Higher Education, 31(3), 299-318.

Eraut, M. (2000). Non-formal learning and tacit knowledge in professional work. British Journal of Educational Psychology, 70, 113-136.

Falk-Ross, F. C. (2002). Classroom-based language and literacy intervention: A programs and case studies approach. Allyn \& Bacon.

Fernández, G. M. E. y Carlino, P. (2006, 28-30 de noviembre). Leer y escribir en la Escuela Media y en la universidad. Diferencias percibidas por ingresantes a la Facultad de Ciencias Humanas de la UNCPBA [ponencia]. XIII Jornadas de Investigación y Segundo Encuentro de Investigadores en Psicología del Mercosur. Buenos Aires.

Fernández, G. y Carlino, P. (2010). ¿En qué se diferencian las prácticas de lectura y escritura de la universidad y las de la escuela secundaria? Lectura y Vida, 31(3), 6-19.

Glaser, B.G. (1992). Basics of grounded theory analysis. Sociology Press.

Hermerschmidt, M. (1999). Foregrounding background in academic learning. En C. Jones, J. Turner y B. Street (eds.), Students writing in the university: Cultural and epistemological issues (pp. 6-16). John Benjamins Publishing. https://doi.org/10.1075/swll.8.06her

Hernández Sampieri, R., Fernández-Collado C. y Baptista Lucio, P. (2008). Metodología de la investigación. McGraw-Hill.

Lea, M. (2005). Communities of practice in higher education: Useful heuristic or educational model? En: D. Barton and K. Tusting (eds.), Beyond communities of practice: Language power and social context. Learning in doing: Social, cognitive and computational perspectives (pp. 180-197). Cambridge University Press.

Lea, M. R., y Street, B. V. (1998). Student writing in higher education: An academic literacies approach. Studies in Higher Education, 23(2), 157-172.

Lillis, T. (1999). Whose common sense. Essayist literacy and the institutional practice of mystery. 10.1075/swll.8.13lil

Lillis, T. (2001). Student writing: Access, regulation, desire. Literacies. Routledge.

Lillis, T. y Scott, M. (2007). Defining academic literacies research: Issues of epistemology, ideology and strategy. Journal of Applied Linguistics, 4(1), 5-32.

Murga de Uslenghi, M. M., Padilla de Zerdán, C., Douglas de Sirgo, S. y Ameijide, M. D. (2002). Discurso estudiantil: representaciones acerca de las competencias discursivas. Análisis crítico del discurso estudiantil en el nivel universitario. Oralidad, lectura y escritura: categorización de dificultades. Revista de Investigaciones Linguisticas y Literarias, 15, 1-9. 
Padilla, C., Ávila, A. M., Douglas, S., García, C., López, E. y Ocampo, M. (2005, septiembre). Taller de comprensión y producción textual: un espacio para leer y escribir géneros académicos [ponencia]. Actas del I Congreso Nacional de Estudios Comparados en Educación. Buenos Aires.

Padilla, C., Ávila, A. y López, E. (2007). ¿Cómo preparamos a los estudiantes universitarios para abordar textos académicos? Lectura y escritura: caminos para la construcción del mundo. Editorial Científica Universitaria de la Universidad Nacional de Córdoba. Cátedra Unesco.

Padilla, C., Douglas, S. y López, E. A. (2010). Argumentative skills in academic literacy. Revista d'innovació educativa, 4, 1-12.

Padilla, C., Douglas, S. y Lopez, E. (2014). Investigar, argumentar y escribir en la Universidad: recorridos dialécticos en estudiantes de Humanidades [ponencia]. xIv Congreso de la Sociedad Argentina de Lingüística (SAL). Palabra, pensamiento, mundo: de la lengua a la interculturalidad. Coloquio de la Cátedra Unesco, Catamarca, agosto del 2014.

Street, B. (2003). What's "new" in new literacy studies? Critical approaches to literacy in theory and practice. Current Issues in Comparative Education, 5(2), 77-91. 